\title{
FROM LAND-USE, REGULATIVE PLANNING TO STRATEGIC SPATIAL VISIONS A LONG AND DIFFICULT TRANSITION IN ITALY
}

\section{A B S T R A C T}

The national planning tradition in Italy dates back to the ' 40 . This article studies the evolution of the discipline in the last seventy years, highlighting the process that has evolved from a strict hierarchic system based on regulative land-use planning and national competencies to regional and local diversification highlighting the new attempts at striving to more strategic spatial visions. 
Italy is a country of several contradictions. It has about 60 million inhabitants who speak a dozen of legally recognised local languages and countless dialects; it stretches from the Alps to the Mediterranean; it belongs to the eight biggest economies in the world but has a very high number of protected natural areas; it celebrates this year its 150 anniversary of the national unification but its history dates back long before the Romans and the remnants are to be found in most of its cities and villages; it is split up in 20 regions, about one hundred provinces and more than 8.000 municipalities each of them responsible at a different scale for planning and development. It is the country with the highest number of UNESCO heritage sites in the world but in which abusive buildings are still an endemic, unresolved issue. Planning and governing spatial development under such circumstances is not easy.

\section{A HISTORIC OVERVIEW}

The national planning system was for the first time defined in the 1942 Town Planning Act (the Planning Law 1150/42). Conceived by the "rationalist" wing during the Fascist period, the proposed planning system was a hierarchical one, based on the strict control of urban and regional spatial development and on direct action by the national government. This was a clear system, based on two types of plans: an urban land-use plan and a regional strategic scheme, the territorial co-ordination plan.

Such plans corresponded to two institutions: the municipalities and the national government. The most widespread level of planning has always been the land-use plan. It provided a framework of policies as well as a detailed zoning scheme, and major normative indications on what to do in each zone. It ensured development rights and building regulations. The most important amendments to the Town Planning Law of 1942 was the so called "Bridge law" of 1967 and the Joint Ministerial Decrees of 1968 introducing mandatory taxes to provide land for public uses and standards for allocating public services and infrastructure in each land-use plan according to the expected population growth over a decade.

It was between the ' $70 \mathrm{~s}$ and the early ' $80 \mathrm{~s}$ that the first stage of planning activity began. It sought to apply the new regional laws and to approve their town-planning instruments. In fact, the first regional territorial plans date from that period. Planning of the 8.103 Italian municipalities is still entrusted to an instrument that has quite different features from those used elsewhere in Europe. In fact the 1942 land-use plan regulates the land-use of the entire municipal territory. In terms of zoning it controls heights, densities, typologies, 
precisely defining buildings to be conserved, as well as the location of services and infrastructure. It is, in effect, a rigid instrument, except that, it is subject to modification processes. Today the land-use plan is acknowledged as inadequate to address the present urban complexity and to respond to the speed of global development. Undoubtedly effective in the period of urban expansion, today it appears far less effective for guiding urban and spatial transformations.

The legal planning system in Italy is now almost 70 years old. The reform process advanced progressively over the past 40 years in a time span significantly greater than that which was necessary to set it in motion.

One can basically speak of three "regionalising" waves considering that governing spatial planning at the national central level could not adequately respond to the various local issues:

- The first was in the '70s, implemented by a partial assignment of functions from the national government to the regional ones. Among these, for the first time, there were administrative functions in the town planning sector, which until that moment had been exercised by the peripheral organs of the central government.

- A second "regionalisation" delegated to the government to implement the regional system. This delegation was developed in a decree which established 15 Regions (five border Regions in the North and the South of the country had already special statutes and greater autonomy).

- The third "regionalisation" wave started in 1997 affirming the principle of subsidiary for all institutional levels and granting further devolution of powers towards local autonomies. Few functions were left to the central government while everything else not thus defined was the task and the function of the regional governments and the local authorities.

Over the past twenty years new regional and national legislation in Italy attempted to experiment in actions of integrating formal rules and real practice. A planning model based on a rigid institutional and policy hierarchy is giving way to the one that recognises the increased autonomy of a variety of stakeholders.

Many things have been changing very quickly. A reform, which opened up long discussions but was never finally approved, is implemented de facto by innovative regional laws and by experiments introduced into many plans in the form of sustainability, of equalising compensation and of more strategic visioning instead of strict land-use planning. 
Such transition, which is still largely experimented with, suffers from uncertainty partly deriving from the delay of national law makers on the issues of land use regime, simpler and more faire taxation, and the application of property rights compensation models to planning.

\section{REGIONAL PLANNING}

The pressure for reform which generated the regional planning laws over the past two decades has broader-ranging effects. New, far stronger and more autonomous regions began to take shape, often with the drive towards total devolution and lately towards federalism. They possess numerous instruments to reshape their institutional identity. These range from the new regional statutes to the new regional laws encompassing a variety of competences. With this strong impulse for legislative innovation, in the last two decades the regions expressed different viewpoints leading to the promulgation of:

- comprehensive, innovative planning laws, combining the novelties deriving from the application of the Law 142/90 (establishment of provincial planning) with innovations in plan forms (especially the municipal ones);

- comprehensive, but not innovative planning laws, which are limited to applying the Law 142/90 but without introducing other elements of innovation. Some organic but not innovative regional Planning Laws have been enacted. Significantly, these are regions (and provinces) with special statutes; they are small and have very specific planning traditions;

- a number of partial laws which address very specific fragments of necessary innovation, at times also underlining important effects regarding plan contents and their effectiveness.

In terms of future intentions, almost all regions, in various ways and at various times, aim at a new organic regional Planning Law. Some are effectively working in that direction, whereas others are contradicting themselves by not carrying out their stated goals.

The result of this process is a chaotic situation when addressing planning issues and even planning jargon both at the regional and local level. A variety of instruments, processes and norms were introduced making the cacophony quite big and making regional autarchy even bigger. Just in the last years of this new millennium the necessity of more comprehensive strategic planning arose between various regions, leading to some new, still ongoing forms of territorial cooperation. 
Today regional planning shows a nation divided into two parts differing one from the other more than from provincial and municipal planning. The country's North and Centre have an appreciable number of territorial plans. The South is still partly without general instruments and is characterised by a stalemate where some experiments are well concluded technically but have not been transformed into current plans. Numerous north-central regions have general policy regional territorial plans. The majority of them were drafted in the '90s. Many regions have reformed their planning legislation, introducing participatory and co-operative actions into planning practice. The result is a widely articulated ensemble of regional instruments bearing witness to an on-going renewal process, which is still evolving. A limitation is represented by the excessive number of plans for the same territory lacking coherent harmonisation, by the scarce integration between economic and territorial planning (an integration that ought to clarify responsibilities, resources and terms) which lack monitoring and control of the results of the actions undertaken.

A special mention about the protection of natural sites also needs to be made. The experience in planning natural parks and other protected areas is decidedly well developed. Today slightly more than $11 \%$ of the natural environment is subject to specific protection measures (generic protection or hydro-geological restrictions). Some of these areas have also been declared Natura 2000 areas following the Habitat Directive (92/43/EU). Furthermore, the portion of land significantly influenced by park policies is far greater, having been estimated as lying between 20 and $25 \%$.

About two-thirds of Italian parks (a percentage not very different from the European one) have a plan. This figure questions the hierarchy of the planning process according to institutional competencies (Regions, Provinces, Mountain Communities and Municipalities). Park planning can substitute, to all intents and purposes, any other form of planning and has all but a sectoral character. A second observation concerns the emergence of a co-operative trend in management and planning. Several Italian experiences combine park policies and local development policies and expand actions to include neighbouring areas. Alongside the traditional "regulatory" mission, parks tend to combine preservation with development choices.

In 1999 a special advisory committee of the Ministry of Environment produced a document regarding the "Guidelines for Environmental and Territorial Policy" which has shed some light on the difficulties, as well as the prospects, for an integrated vision. 
General guidelines would integrate environmental and territorial policies going beyond political attitudes and cultural traditions that have trapped protection actions in the defence of monuments, nature reserves, and threatened species while granting public works and urban development the guiding action. This "trespassing" seems to impose itself whether one begins with the territory or from the issues such as defence of biodiversity that are closely tied to the management of the economic-territorial diversification processes. It is demonstrated that effective environmental protection depends amply upon spatial planning and territorial policies. A new philosophy of territorial government seems to be surfacing, renewing the way the consideration of hydrological resources influences the planning framework.

Following the last years' (d)evolution in spatial planning and considering that strict regulative land-use planning is no more able to tackle today's society needs, considering also that administrative boundaries very often hinder a correct overview of a territory's development, taking finally into consideration the experiences derived from EU cooperation programmes towards territorial cohesion, some experiences were started over the last few years both at the local and at the regional level.

In 2007 the North Italian regions decided to cooperate together in order to achieve more insight and coordinate their respective spatial development policies.

The whole process was started informally, involving regional department responsible for spatial planning and environmental conservation, but also bringing together the regional political representatives to share the work done at the technical level.

The main aims were to exchange experiences and best practices in spatial development issues, to develop shared visions which would be embedded in the respective regional planning schemes and programmes, to cooperate in drafting calls for EU programmes, to exchange knowledge on particular themes (ecologic networks, infrastructural nodes, territorial capital, etc.), to define a common jargon referred to the various planning instruments and to raise awareness on common problems and strengths and potentials of the territories involved.

Three years of work made it possible to set up an Interregional Working Party, which elaborated visions for the whole northern part of the country showing its strengths and its vocations within a wider European context. 
The aim of the interregional working party was to strengthen cooperation so as to facilitate territorial integration and to promote more competitive and sustainable forms of territorial development than would result from individual, segmented territorial development policies. The work is still progressing, but has clearly shown the importance of cooperation among regions which also resulted in sharing the intents at the political level.

\section{MUNICIPAL PLANNING}

Twenty years after the national Law 142/90 was enacted, the overall picture is a composite one reflecting regional attitudes on the subject of provincial planning; where the first normally prevails over the latter. The coincidence between the provincial schemes and the renewal of planning legislation in some regions fostered innovation. A strongly differentiated national picture results from the provincial planning and regional achievements on landscape and environment legislation, albeit with delays in some southern regions. However, two other fundamental issues seem unresolved:

- the question of mobility and traffic, the contemporary city's prime question, has not been resolved by acting exclusively upon infrastructure provision $y$, but also on the demand for mobility and therefore, in the final analysis, on the city's very form and quality. Spatial development schemes are often lacking;

- the weak relationship between spatial and economic planning: plans are limited to environmental protection and road layout, and often lack any economic integration.

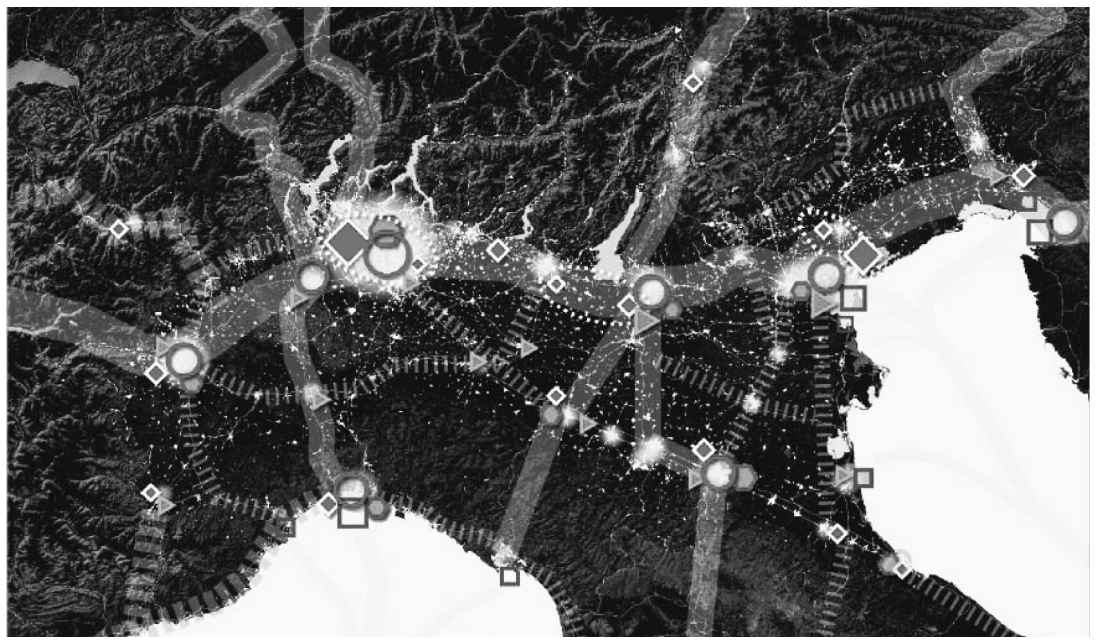

Figure 1 Tavolo Interregionale per lo sviluppo dell'area Padano-Alpina - Vision 2008 (Infrastructure) The illustration of the interregional development of Padano-Alpina area 
Already in 1995 almost all Italian municipalities (96,4\%) had a general town planning instrument (land-use plan) and as many as eight of the regions had total planning coverage of their territory. Two regions with only $78 \%$ of their municipalities possessing a plan, represented the less "covered" situations. Nowadays this gap has almost been covered. Most of the municipal land-use plans date back to the ' 60 and the ' 70 , these have been updated almost every decade. In fact the legislative framework indicates compulsory plan renewal every ten years, i.e. an average of $10 \%$ of the territory (or of the population) planned annually. Aside from some apparently surprising data which should be considered in relation to historic gaps (almost everywhere in the South it is a question of the first or the second generation planning, whereas in the North it is the third, or even the fourth, generation), it appears clear that, even in the best cases, the theoretic figure for ten-year renewal is far lower than the concrete possibilities.

Since 1995 planning activity has involved, in one way or another, more than 2.000 municipalities, or $25 \%$ of the whole. This is an interesting element due both to its global dimension (about 350-400 new municipal plans every year) as well as to a trend which has progressively increased. For the ten-year renewal process there is also the possibility to amend plans as required at any time. This habit has led in some parts of the country to various modifications to the planning instrument, thus sometimes distorting the original planning goals and development perspectives.

Alongside the traditional land-use plans based on more or less rigid regulative structures and on mere quantitative growth indices, some others introduced and applied a dual instrument. According to this original approach, the general instrument becomes a sort of Framework Document for Planning Policies. Its purpose is to define both the administration's municipal strategies to guide public and private policies and projects as well as conditions for assessing development proposals. The framework document flexibility regarding land use is ensured by the approval of operational plans also to be activated by private stakeholders under the framework document.

Quite recently new strategic forms of local and town planning have been experimented all over the country. Aiming at sustainable development and territorial cohesion requires the preparation of plans beyond the local level. Without general agreement about basic ideas and objectives, plans can hardly become effective. This is a big challenge for planning practice, since society as well as public organisations are increasingly fragmented. Therefore, effective planning requires the organisation of consensus building processes; 
this implies at least, open interactive involvement to overcome different views towards objectives, conflicting interests, competing competencies, legal restrictions.

The need to respond quickly to the issues posed by economic and social development imposes having clear views of a territory's future but also enough flexibility for its implementation. The existing local planning instruments show their weaknesses in various circumstances: they are too formal and procedures are normally quite bureaucratic; they do not allow community involvement not even when decisions are already taken and people can eventually appeal; they do not facilitate common understanding and support of a territory's development perspective; they are too strictly bound by administrative borders and sectoral approaches; they do not always take in equal consideration economic, social, cultural and environmental factors fordevelopment (a territory's capital) and hardly evaluate different scenarios. All this leads very often to amending the plan quite often in order to adjust it to new upcoming public or private interests.

Over the course of the last few years, territorial governance all over the country became enriched with the new instruments at the municipal level, manifesting the widespread trend to draw up first-time solutions to new questions, or to long-lived issues for which traditional instruments have proved to be ineffective. Despite the sometimes improvised character of these experimentations, it is possible to find out some recurrent elements that might help to identify long-term trends that are bound to influence the evolution of Italy's planning system.

There is great and variable effervescence of administrative actions and initiatives of various sorts which contribute significantly towards spreading a "new culture" of intervention in Italy. Hence this contributes towards modernising and often reorganising administrative practices.

Some of these attempts were derived from the experiences gained while participating in EU programmes like Interreg or in Local Agenda 21 actions and the key factor of success of some of them relies on not yet being rigidly and rigorously regulated. They encompass all planning scales whether or not targeted at physical transformations or related to urban transformations. However, it is at the local level where this trend to move away from regulative planning towards a more strategic, integrative approach is felt to be of utmost importance. 
The complexity of policies for local development lies in balancing actions seeking economic, social, environmental and cultural development. Thus, their salient feature is involvement of the private sector, not just in financial terms, but in terms of participation and of joint responsibility in the local administration's decisions. Moreover, it is evident that this kind of approach profoundly modifies the historic role of the (local) public administration from being the decision-maker to being the promoter or the facilitator; and this involves equally plainly an administrative culture that is very different from what previously was quite usual in Italy, and also - and this aspect should not be underestimated - a technical and professional contribution that is demanding high and specialised competencies next to the ability to work in large teams and to face issues in a holistic way.

One of the most recent experiences of a spatial planning process aiming at consensus building among a large variety of stakeholders and interests, and working as good as possible towards a common spatial vision is the Gardena Masterplan. Gardena is a valley in the North of the country, a tourist destination for both winter and summer holidays. This small valley, comprising five municipalities and about 15.000 inhabitants who belong to three differently speaking ethnic groups, has undergone a quick development over the last 20 years. Income rates in the valley are among the highest in the whole region, but this also causes an increase in the costs of dwellings due to the high demand generated by tourists and because of scarcity of land. The valley also has the accessibility problems, due to its physical structure, with the result that during high season periods the main road is very often congested.

Two years ago the mayors decided it was time to join efforts and work together towards common goals and solutions. Each of them had always planned its own territory without caring too much about what others did or needed. Traffic problems, congestion, air and water ollution, noise, location of main infrastructure, erosion of natural habitats and detriment of cultural values affected the entire valley; these issues needed to be jointly tackled. With the support of the regional departments an informal process towards a common spatial vision was initiated. The goal was to identify common local potentials and key factors of development and elaborate scenarios for the future.

The aims were to achieve consistency of the different approaches and in the long term, to have more coherence between targets and solutions, to be creative, to involve all relevant parties and stakeholders in a real bottom- 
up process, to connect the various parts of a unique territorial system which should be seen as a functional unit.

The Masterplan Gardena was characterised by these different steps:

- the process towards a spatial vision was started informally,

- local key factors for development were identified,

- the relevant territory was defined regardless of any administrative borders,

- stakeholders (representing valuable economic, ecologic, social and cultural interests) were invited to participate actively in SWOT analysis, - community involvement was organised to formulate main goals and targets. People rightly wanted to fully understand the proposals and recognise their ideas and interests,

- different scenarios were elaborated and evaluated,

- an agreement on a preferred solution (common vision) was taken by the local population organised in forums,

- the legal framework and action plans supporting the decision-making process and the implementation were elaborated.

Now the implementation phase should start. Local land-use plans will be amended accordingly and actions or decisions will need to be coherent with the overall goals set in the Masterplan. Legal plans will remain a tool for managing the globally agreed future spatial development.

In Italy the evolution of the discipline, the various institutional arrangements and the increased subsidiarity in planning resulted in different legal procedures with regard to the management of territories in the various regions. As a result, planning practice varies nowadays considerably throughout the country; in some parts the focus is still anchored on regulating land-use on the local level, in other parts the approach is broader, focussing on strategic future developments and on territorial cohesion and cooperation. Various planning cultures are emerging.

Although attempts to modernise procedures, instruments and governance are based on the increased awareness that the "territory matters" and that more integrated approaches are required, modern planning in Italy has still a long way to go. 
Correia P., Bussadori V., Griffith J., Papayannis T., Vogelij J., (2003) The New Charter of Athens 2003, the European Council of Town Planners Vision on the City of the 21st century - Alinea Florence 2003

INU (2000); Report on the State of Planning, Rome 2000

Vogelij J. (2008); Strategic Planning towards Territorial Cohesion, ECTP-CEU website

Vogelij J. (2010); The Role of Concepts for the Effectiveness of Decision Making in Some European Spatial Planning Processes, Soest 2010 